\section{Scientific Life Fueling the Bio-economy: European Culture Collections and Microbiology Education and Training}

André Antunes, ${ }^{1,2,4, *}$ Erko Stackebrandt, ${ }^{3}$ and Nelson Lima ${ }^{1}$

A survey of European Microbial Biological Resource Centers and their users provided an overview on microbiology education and training. The results identified future increases in demand despite several shortcomings and gaps in the current offer. Urgent adjustments are needed to match users' needs, integrate innovative programs, and adopt new technologies.

\section{Microbial Diversity and} Microbiological Resource Centers The abundance and diversity of microbes is breathtaking, as emphasized in multiple studies. For example, current estimates of the total number of bacterial cells on Earth $\left(4-6 \times 10^{30}\right)$ outnumber the estimated number of stars in the universe by several orders of magnitude $\left(10^{21}\right)$ [1]. Microbes are incredibly resilient and successful, populating all sorts of different environments, including several inhospitable ones previously thought to be sterile (e.g., healthy placentas [2], black smoker fluids in hydrothermal vents [3], deep-sea brines [4]). They dominate not just our oceans, terrestrial sites, and deep subsurface environments, but also our own bodies: total counts show that bacteria associated with the human body thrive and outnumber our own cells and genes at 10:1 and 100:1 ratios, respectively $[5,6]$.
Due to their enormous genetic and functional flexibility, microbes have a wide impact on our planet. Microbes, either being beneficial or detrimental, play essential ecological roles, interacting with plants and animals, and control vital global geochemical and nutrient cycles. Humans have been making direct use of microbes since the dawn of humankind - originally associated with the production of fermented foods and beverages, but during the past half century as producers of antimicrobial agents and enzymes for application in various areas of modern-day biotechnology (e.g., for medicines, fuel production, farming and the food industry, forensics, and bioremediation (http:// www.oecd.org/science/biotech/ 1890904.pdf).

Public microbial culture collections and the quality-managed Microbial Biological Resource Centers (mBRCs) fuel the BioEconomy: they preserve biodiversity, and provide access to authenticated microbial resources and to associated data and expertise. Future innovation in biotechnology heavily depends on the use of microbial resources as raw materials, as well as on access to knowledge and expertise in the various disciplines within microbiology.

MIRRI (the Microbial Resource Research Infrastructure: www.mirri.org) is a European Union project as part of the ESFRI initiative by the European Council (European Strategy Forum on Research Infrastructures: http://www.esfri.eu/). MIRRI involves 16 partners in 11 European countries and 27 Collaborating Parties in 8 other countries, collectively holding over one million microbial strains, plasmids, viruses, and DNA samples. This project, which is currently at the end of its preparatory phase, aims to solve the longstanding issues of fragmentation and overlap in holdings, services, and education and training (E\&T) offered by mBRCs. In general, it will allow coordination between the different mBRCs and create a pan-European distributed research infrastructure that will ensure harmonized broadening of holdings and connect resource holders with end-users more efficiently, thus, promoting knowledge transfer and fostering innovation [7-9].

One crucial element towards achieving these goals is to properly define the user community, and identify trends in the current and future demand for services, as well as possible gaps and bottlenecks. A survey was performed to identify these and is discussed below together with specific recommendations.

\section{Education and Training}

\section{Requirements in Microbiology}

A questionnaire-based survey was established, targeting current and potential users of microbial resources and services and aiming to identify trends in demand for all services provided by mBRCs, as well as possible gaps [10]. From a total of 1146 individual users, 998 replies (758 from the non-profit sector, and 238 from the forprofit sector) were collected and analysed on the basis of E\&T.

Despite the widely recognized importance of E\&T in technology transfer and innovation, only a low number of respondents accessed E\&T from any external sources. Merely $17.9 \%$ of respondents outsourced E\&T in the past 5 years, while even fewer (15.9\%) intend to do so in the next 5 years. The differences between the for-profit and non-profit sectors were minor, although, for the latter, demand for the next 5 years is expected to remain stable. The scenario for E\&T outsourced to mBRCs looks somewhat positive as increases in demand are expected for the next 5 years, particularly in the for-profit sector with $5.8 \%$ growth.

Multiple factors were behind the low use of the E\&T offered in microbiology by mBRCs (Figure 1). While some stated that E\&T are not needed and/or are taken care of in-house, others pointed to the lack of visibility of $\mathrm{mBRC}$ teaching and training and other shortcomings (particularly on 
(A)

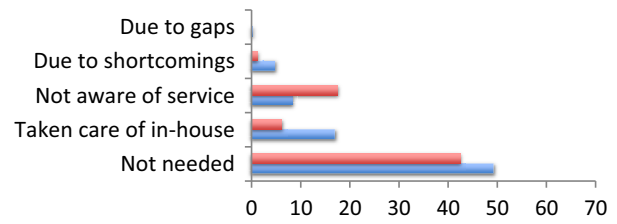

(B)

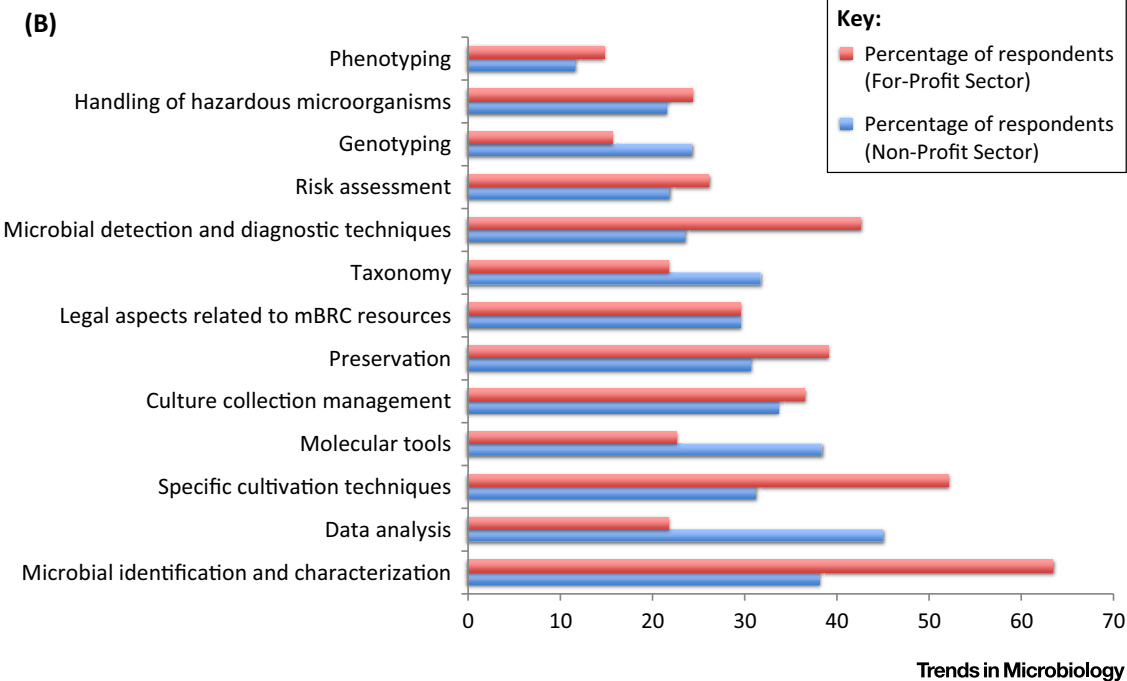

Figure 1. Overview of Reasons Invoked for Not Making Use of Microbiological Training Provided by Microbial Biological Resource Centers (mBRCs) (A), and Top Training Topic Needs Identified by For-Profit and Non-Profit Sectors (B).

price competitiveness). Despite the presence of a wide E\&T offer to users, they were listed as the second highest service that repliers were 'not aware of' as being provided by mBRCs, a trend particularly pronounced in the for-profit sector.

We also observed that the list of E\&T topics identified in replies by $\mathrm{mBRC}$ users is markedly different when comparing the for-profit and the non-profit sectors. Remarkably, the highest rated topics by respondents from the for-profit sector are much more aligned with the classic core activities of mBRCs (e.g., microbial identification and characterization, microbial cultivation, and preservation). Respondents from the non-profit sector (predominantly in academia) are likely better suited to address such issues in-house, having higher demand for topics associated with higherend technologies and tools (e.g., data analysis, molecular tools). Such differences reflect the specificity and differentiation in needs and skills required and available in academia and industry (Figure 1).

\section{Current Offer in E\&T in} Microbiology Provided by MIRRI mBRCs

An additional survey targeting mBRCs within the MIRRI consortium was designed and circulated. Replies from 28 mBRCs across Europe (almost all of the MIRRI mBRCs) provided a snapshot of current education and training, tools, and contents being used and produced within the consortium, identifying areas that need improvement. The scenario revealed by this second survey needs special attention.

E\&T still relies almost exclusively on faceto-face interaction (only one partner makes use of combined face-to-face and online interaction, that is, b-learning or blended learning). Likewise, and despite the current trends in increased use of new formats for E\&T [11-14], current offerings by MIRRI mBRCs are still overwhelmingly dominated by a traditional lecture-centered style with support of textbooks or print materials (only one-third of MIRRI mBRCs make use of video, interactive, or other types of new learning resources, and only about a quarter of MIRRI mBRCs produce them).

It is also interesting to note that some mBRCs within MIRRI do not offer E\&T as part of their services. This is usually due to a lack of personnel and/or financial resources. However, most collections recognize the need to expand E\&T as part of an improved offer to users. The set of innovative tools available will permit the integration, modernization, and harmonization of the MIRRI E\&T offerings and fill the identified gaps, by developing and designing new approaches to deliver E\&T and supporting the improvement of the current offerings. Specifically, it is recommended to: (i) bridge the current gap between E\&T offerings and demand by implementing new courses either in continuing professional development (CPD) schemes or postgraduate (masters and $\mathrm{PhD}$ ) degrees offered with higher education institutions; (ii) increase awareness of E\&T offerings by advertising on home websites and on course aggregators; and by running massive open online courses (MOOCs) on a selection of general topics; (iii) increase course attendance by widening offerings to prospective trainees in remote locations via online accessible contents using small private online courses; (iv) improve E\&T quality by constant content update and review; (v) contribute to development of a portfolio-based professional culture that will permit the development of unique profiles of skills and capacities in jobs for the future, through the flexibility, comprehensiveness, and accreditation of E\&T offerings (as discussed by multiple research infrastructures, namely within, for example, the LifeTrain and EMTRAIN projects [15]); and (vi) create trust on the MIRRI label and shift towards bioindustry needs.

The mBRCs' role in underpinning future biotechnology breakthroughs should also rely on knowledge transfer on topics such 
as new cultivation methods for less common microbial groups, or microbial identification with integration of new cutting-edge technologies (e.g., next-generation, singlecell, and whole-genome sequencing as well as MALDI-TOF MS). These key E\&T topics have not been fully explored and can accelerate the development of new bioproducts and services. Such a role for mBRCs reflects the central position of research infrastructures in innovation in new technologies, and a privileged role in training researchers in how to make the most of such new advances and technologies.

\section{Concluding Remarks}

mBRCs are key holders of microbiological resources, data, and expertise which are crucial for research and innovation. Furthermore, E\&T is one of the main paths for knowledge transfer between mBRCs and users of microbiological resources in academia and, most importantly, in industry. Nonetheless, this service has received insufficient attention, and has been hampered both by access to limited resources and the absence of information on present and future needs and demands, which has never been previously collected.

There is a much wider market available in the field of E\&T, and the demand is likely to increase in the future. mBRCs still rely on outdated methods and tools for E\&T, and are clearly underprepared to face this challenge. Further efforts are clearly required in adjusting E\&T offer, adapting contents and content delivery whilst focusing on cost-efficiency and efficient advertising to increase visibility. MIRRI's ongoing efforts in this field will facilitate: (i) the pooling of resources, and (ii) the coordination of training content production, courses offered, and their advertisement. Adopting e-learning, b-learning, video, and interactive content will be particularly beneficial due to scalability, and the production of reusable and 'mashable' content $[11,14]$. Also, this will increase the reach and accessibility of E\&T courses, reduce unnecessary face-to-face components, optimize course duration, and reduce costs to $\mathrm{mBRC}$ and end-users. Such improvements will contribute significantly to the sustainability of mBRCs.

Improving the current E\&T offered by mBRCs is a complex task, but an essential one if we want to increase its quality and effectiveness, improve their alignment with the needs of end-users, and thus assist in fueling the current and future waves of innovations in biotechnology.

\section{Acknowledgments}

This project has received funding from the European Union's Seventh Framework Programme for research, technological development and demonstration under grant agreement no 312251. The work presented here would not have been possible without the dedicated efforts of all members of the MIRRI consortium, particularly the teams in Work Package 2 and 7.

${ }^{1} \mathrm{CEB}$ - Centre of Biological Engineering, Micoteca da Universidade do Minho, University of Minho, Braga, Portugal

${ }^{2}$ King Abdullah University of Science and Technology (KAUST), Computational Bioscience Research Center (CBRC), Building 3, office 4216-WS01, 4700 KAUST, Thuwal, 23955-6900, Saudi Arabia

${ }^{3}$ Leibniz Institute - German Collection of Microorganisms and Cell Cultures GmbH, Braunschweig, Germany ${ }^{4}$ Current address: Department of Biology, Edge Hill University, St Helens Road, Ormskirk, Lancashire L39 $4 \mathrm{QP}, \mathrm{UK}$

*Correspondence: andre.antunes@kaust.edu.sa, antunesa@edgehill.ac.uk (A. Antunes).

http://dx.doi.org/10.1016/j.tim.2015.11.010

References

1. Whitman, W.B. et al. (1998) Prokaryotes: the unseen majority. Proc. Natl. Acad. Sci. U.S.A. 95, 6578-6583

2. Aagaard, K. et al. (2014) The placenta harbors a unique microbiome. Sci. Transl. Med. 6, 237ra65

3. Takai, K. et al. (2008) Cell proliferation at $122^{\circ} \mathrm{C}$ and isotopically heavy $\mathrm{CH}_{4}$ production by a hyperthermophilic methanogen under high-pressure cultivation. Proc. Natl. Acad. Sci. U.S.A. 105, 10949-10954

4. Antunes, A. et al. (2011) Microbiology of the Red Sea (and other) deep-sea anoxic brine lakes. Environ. Microbiol. Rep. 3, 416-433

5. Bäckhed, F. et al. (2005) Host-bacterial mutualism in the human intestine. Science 307, 1915-1920

6. Savage, D.C. (1977) Microbial ecology of the gastrointestinal tract. Annu. Rev. Microbiol. 31, 107-133

7. Schüngel, M. and Stackebrandt, E. (2015) Microbia Resource Research Infrastructure (MIRRI): Infrastructure to foster academic research and biotechnological innovation. Biotechnol. J. 10, 17-19

8. Smith, D. et al. (2014) Investment into the future of microbial resources: culture collection funding models and BRC business plans for biological resource centres. Springerplus 3,81

9. Stackebrandt, E. et al. (2014) Deposit of microbial strains in public service collections as part of the publication process to underpin good practice in science. Springerplus 3, 208
10. Stackebrandt, E. et al. (2015) The microbial resource research infrastructure MIRRI: strength through coordination. Microorganisms 3, 890-902

11. Radović-Marković, M. (2010) Advantages and disadvantages of e-learning in comparison to traditional forms of learning. Ann. Univ. Petroșani Econ. 10, 289-298

12. Redecker, C. et al. (2011) The Future of Learning: Preparing for Change. European Commission Joint Research Centre Institute for Prospective Technological Studies EUR 24960 EN Luxembourg, Publications Office of the European Union

13. Johnson, L. et al. (2014) NMC Horizon Report: 2014 Higher Education Edition, The New Media Consortium

14. Waldrop, M.M. (2013) Education online: the virtual lab. Nature 499, 268-270

15. Hardman, M. et al. (2013) LifeTrain: towards a European framework for continuing professional development in biomedical sciences. Nat. Rev. Drug Discov. 12, 407-408

\section{Forum}

Septal Junctions in Filamentous Heterocyst-Forming Cyanobacteria

\author{
Enrique Flores, ${ }^{1, \star}$ \\ Antonia Herrero, \\ Karl Forchhammer, ${ }^{2}$ and \\ Iris Maldener ${ }^{2}$
}

In the filaments of heterocyst-forming cyanobacteria, septal junctions that traverse the septal peptidoglycan join adjacent cells, allowing intercellular communication. Perforations in the septal peptidoglycan have been observed, and proteins involved in the formation of such perforations and putative protein components of the septal junctions have been identified, but their relationships are debated.

\section{The $\mathrm{N}_{2}$-Fixing Cyanobacterial Filament}

Some cyanobacteria grow as chains of cells (filaments or trichomes) that can be hundreds of cells long. The cyanobacteria have a Gram-negative-type cell envelope, and the cyanobacterial filament consists of individual cells surrounded by their peptidoglycan layers but enclosed in a 